\title{
Herausforderungen an eine zeitgenössische feministische Menschenrechtspolitik am Beispiel sexualisierter Kriegsgewalt ${ }^{1}$
}

Feministische Forschung kritisiert seit langem einen gender bias des internationalen Rechts in allen seinen Bereichen. ${ }^{2}$ Im Bereich der Menschenrechte und des internationalen Strafrechts ist ein zentrales Thema dieser Kritik die unzureichende Einbeziehung sexualisierter Gewalt gegen Frauen. Die Mobilisierung unter dem Slogan »Frauenrechte sind Menschenrechte« und nicht zuletzt die intensive Medienberichterstattung über die systematische sexualisierte Gewalt gegen muslimische Frauen in Bosnien und die darauf folgenden Anklagen vor dem Jugoslawien-Tribunal (ICTY) haben das Problem von Vergewaltigungen als Mittel der Kriegsführung international auf die Tagesordnung gesetzt. Dennoch hat das Thema sexualisierter Kriegsgewalt nicht an Aktualität verloren, wie sich im Kongo zeigt. 3 Gleichzeitig haben Theorieentwicklungen in der Geschlechterforschung neue Fragen formuliert, die den politischen Ansatzpunkt des Konzepts »FrauenMenschenRechte« herausfordern.

\section{Feministische Errungenschaften: Anerkennung geschlechtsspezifischer Menschenrechtsverletzungen auf der internationalen Ebene}

Charlesworth, Chinkin und Wright gelten mit ihrem Aufsatz von I99 I als Begründerinnen der feministischen Kritik am internationalen Recht. 4 Im Zentrum dieser Kritik steht die dichotomische Struktur des internationalen Rechts, insbesondere der public private split, der sich durch sämtliche Rechtsbereiche zieht's und insbesondere im Bereich der Menschenrechte zu einer Unterbelichtung geschlechtsspezifischer Rechtsverletzungen führte. Menschenrechte beschränkten sich auf die `öffentliche < Sphäre, während das >Private - die physische und sexuelle Reproduktion - der staatlichen Verantwortung weitgehend entzogen war. ${ }^{6}$ Der Bereich des Privaten bedeutete für Männer einen geschützten Rückzugsort, der Freiheit vor staatlicher Kontrolle garantierte, es ihnen jedoch gleichzeitig erlaubte, ungestraft die Freiheit anderer gewaltsam einzu-

I Für Prof. Dr. Susanne Baer, die unseren Denkprozess inspiriert und unterstützt hat.

2 Maßgeblich Hilary Charlesworth/Christine Chinkin, The Boundaries of International Law: A Feminist Analysis, Manchester 2000. Aktuell Doris Buss/Ambreena Manji (Hrsg.), International Law: Modern Feminist Perspectives, Oxford/Portland, Oregon 2005; Beate Rudolf (Hrsg.), Frauen und Völkerrecht: Zur Einwirkung von Frauenrechten und Fraueninteressen auf das Völkerrecht, Baden-Baden 2006; Andreas Zimmermann/Thomas Giegerich (Hrsg.), Gender und Internationales Recht, Berlin 2007.

3 Statement der Sonderberichterstatterin des UN-Menschenrechtsrats über Gewalt gegen Frauen, ihre Ursachen und Wirkungen, Y. Ertürk, vom 27.7.2007, abgedr. in Presseerklärung der UN vom 30.7.2007 (www.onug.ch).

4 Hilary Charlesworth/Christine Chinkin/Shelley Wright, Feminist Approaches to International Law, AJIL 85 (I99I), 6I3-645. Zwar gehen feministische Initiativen im Bereich des internationalen Rechts viel weiter zurück, eine umfassende theoretische Analyse jedoch fehlte.

5 Überblick bei Anne Peters, Völkerrecht im Gender-Fokus, in: Zimmermann/Giegerich (Fn. I), I99-299. Ausführl. Charlesworth/Chinkin (Fn. 2).

6 Vgl. Hilary Charlesworth, Human Rights as Men’s Rights, in: Julie Peters/Andrea Wolper (Hrsg.), Women's Rights, Human Rights: International Feminist Perspectives, New York/London 1995, I03-I I3; Donna Sullivan, The Public/Private Distinction in International Human Rights Law, ebd., I 26-I 34 . 
schränken.7 Das Private der Sexualität erstreckte sich sogar auf die Handlungen von Amtsträgern oder Soldaten, deren Übergriffe als bloß individuelle Triebverbrechen galten.

Seit 199I hat sich gerade im Bereich der Menschenrechte viel getan: »feminism started to get >a seat at the table $<. \ll^{8}$ Einen wesentlichen Beitrag dazu leistete die unter dem Slogan »Frauenrechte sind Menschenrechte « 9 mobilisierte internationale Frauenbewegung. Sie forderte einerseits die Anerkennung frauenspezifischer Rechtsverletzungen, insbesondere Gewalt, als Menschenrechtsverletzung und andererseits ein Mainstreaming der Rechte von Frauen in den existierenden Menschenrechtsbereich auf allen institutionellen Handlungsebenen. ${ }^{\text {IO }}$

Während das Mainstreaming sich nur langsam durchsetzen konnte, ${ }^{\text {II }}$ konnte das Thema Gewalt gegen Frauen, das von der gleichstellungsorientierten UN-Frauenrechtskonvention CEDAW ${ }^{12}$ I 979 nicht ausreichend erfasst war, in diesem Zuge erfolgreich auf die internationale Tagesordnung gesetzt werden. Zum einen wird häusliche Gewalt zunehmend als Menschenrechtsverletzung anerkannt, die ein Staat zu verantworten hat, der durch Untätigkeit diese Übergriffe duldet. Zum anderen stellte die Anerkennung sexualisierter Gewalt als Menschenrechtsverletzung einen Meilenstein dar, indem sie die Trennung von privat/öffentlich auf deutliche Weise überwindet. Vergewaltigungen sind nicht schon aufgrund der Sexualisierung sprivat<, sie werden systematisch zur Folter und als Mittel der Kriegsführung eingesetzt: »Taking the women is taking the land «. Dem entspricht eine Einordnung sexualisierter Gewalt als Kriegsverbrechen, als Folter und als Verfolgungstatbestand im Flüchtlingsrecht. Erst im Mai 2008 wurde Jean-Pierre Bemba, Vorsitzender des Mouvement de Libération du Congo (MDC), wegen Terrorkampagnen gegen die Zivilbevölkerung in der Zentralafrikanischen Republik verhaftet. Der Chefankläger des Internationalen Strafgerichtshofs kommentierte: »Mr. Bemba's arrest is a warning to all those who commit, who encourage, or who tolerate sexual crimes. There is a new law called the Rome Statute. Under this new law, they will be prosecuted. «13

Die feministische Menschenrechtspolitik hat insofern wichtige Erfolge vorzuweisen. ${ }^{14}$ Allerdings sind diese Erfolge prekär und limitiert. Nach wie vor besteht ein paralleles Sonderregime für Frauenrechte, wie es im Monitoring-

7 Dass dieser Schutzraum nur für einige galt, zeigt sich etwa im repressiven Vorgehen gegen gleichgeschlechtliche Sexualität sogar in der Privatheit des eigenen Schlafzimmers; Wendy Brown, Suffering the Paradoxes of Rights, in: dies./Janet Halley (Hrsg.), Left Legalism/Left Critique, Durham/London 2002, $420-434,428$.

8 Thérèse Murphy, Feminism Here and There: Law, Theory and Choice, in: Buss/Manji (Fn. 2), 67-86, 7I.

9 Ursprünglich eine Forderung lateinamerikanischer Feministinnen, vgl. Elisabeth Friedman, Women's Human Rights: The Emergence of a Movement, in: Peters/Wolper (Fn. 6), I8-35, 22; maßgeblich dann Charlotte Bunch, Women's Rights as Human Rights: Toward a Re-Vision of Human Rights, Human Rights Quarterly, I 2 (1990), 486-498.

ı Vgl. die Massenpetition von I991, die Weltkonferenz Menschenrechte in Wien I 993 solle »comprehensively address women’s human rights at every level of its proceedings « Friedman (Fn. 9), $27 \mathrm{f}$.

I I Gender Mainstreaming ist seit 1997 Aufgabe des Assistant Secretary-General and Special Adviser to the Secretary-General on Gender Issues and the Advancement of Women, vgl. http://www.un.org/womenwatch/osagi/gendermainstreaming.htm; eingehend Hilary Charlesworth, Not Waving but Drowning: Gender Mainstreaming and Human Rights in the United Nations, Harvard Human Rights Journal i 8 (2005), I-I8; Sari Kouvo, The United Nations and Gender Mainstreaming: Limits and Possibilities, in: Buss/Manji (Fn. 2), 237-252.

I 2 Convention on the Elimination of all Forms of Discrimination Against Women (CEDAW), BGBl. I985 II, 648 .

I 3 ICC Arrest Jean-Pierre Bemba - massive sexual crimes in Central African Republic will not go unpunished, Presseerklärung v. 24.8.2008, http://www.icc-cpi.int/press/pressreleases/371.html (3.6.2008); Case No. ICC-01/05-01/08-I.

I 4 So auch Sonja Buckel, Feministische Erfolge im transnationalen Recht: Die juridische Aufarbeitung des japanischen Systems sexueller Sklaverei, Leviathan 36 (2008), 54-75. 
System für die CEDAW ${ }^{\text {Is }}$ bereits 1982 angelegt war und etwa durch eine Son-

derberichterstatterin zur Gewalt gegen Frauen ausdifferenziert wurde. Die Berichterstattung über Menschenrechtsverletzungen an Frauen ist durch die institutionelle Selbständigkeit der CEDAW-Kommission vom Bereich der >normalen< (universellen) Menschenrechtsorgane weitgehend isoliert ${ }^{16}$ und schwächer ausgestattet. Die >Frauenfrage $<$ konnte sich überdies bisher nur in den sogenannten »weichen« Bereichen des internationalen Rechts durchsetzen; der Bereich der internationalen Sicherheit ist bisher von dieser Entwicklung weitgehend unbeeinflusst geblieben. Im Bereich des Sicherheitsrechts ist der Bezug auf Frauenrechte allenfalls rhetorisch ${ }^{17}$ und in der Diskussion um »human security I $^{18}$ interventionistisch aufgeladen. ${ }^{19}$ Auch die sexualisierte Gewalt gegen Frauen in der Demokratischen Republik Kongo geschieht trotz der Verhaftungen weiter vor den Augen von UN-Blauhelmen, deren Schutzmandat jedoch in dieser Hinsicht weitgehend ungenutzt zu bleiben scheint, ${ }^{20}$ die sie teilweise gar selbst verüben. ${ }^{21}$ Insofern sind die Erfolge, so bedeutend sie sind, oft oberflächlich, rhetorisch und beschränkt und müssen ständig verteidigt werden. ${ }^{22}$

\section{One Step Bebind? Aktuelle theoretische Herausforderungen an eine fe- ministische Menschenrechtspolitik}

Die politische Verankerung von »FrauenMenschenRechten« wurde begleitet von der zunehmenden Etablierung feministischer Völkerrechtswissenschaft als eigenem Feld. ${ }^{23}$ Diese beschäftigte sich mit der Offenlegung eines »männlichen Paradigmas « im Völkerrecht und der Unterrepräsentanz von Frauen und ihren Interessen. Inzwischen sind jedoch innerhalb der Geschlechterforschung Ansätze wirkmächtig geworden, die gerade die Kategorien >Mann $<$ und >Frau $<$ in Frage stellen. Statt Geschlecht als naturgegebene Identitätskategorie zu fassen, wird Geschlecht als interdependente, binäre und heteronormative gesellschaftliche Struktur gedacht. ${ }^{24}$ Ein Geschlechtsbegriff, der jedoch kein abgrenzbares Menschenrechtssubjekt mehr benennt, stellt sich als Herausforderung für die

Is Ausf. Hanna Beate Schöpp-Schilling, Das Übereinkommen zur Beseitigung jeder Form von Diskriminierung der Frau (CEDAW) und sein Vertragsausschuss nach 20 Jahren - Bilanz und Ausblick, in: Zimmermann/Giegerich (Fn. 2), I37-I69.

I6 Diese ist allerdings seit Anfang 2008 nicht mehr dem DAW (Department for the Advancement of Women), sondern dem UNHCHR (UN-Hochkommissar für Menschenrechte) angegliedert.

I7 Kritisch aus postkolonialer Perspektive Sunera Thobani, White Wars: Western feminisms and the > War on Terror<, Feminist Theory 8 (2007), I69-1 85.

I 8 Grundlegend Barry Buzan, People, States and Fear, Boulder, Colorado, I99ı; zu feministischen Reformulierungen von Sicherheit J. Ann Tickner, Gendering World Politics: Issues and Approaches in the Post-Cold War Era, New York 200I, 6I-64.

I9 United Nations, A More Secure World: Our Shared Responsibility. Report of the High-level Panel on Threats, Challenges and Change, New York 2004, 67, 106, http://www.un.org/secureworld/report2.pdf; zur feministischen Kritik an UNO-Konzepten vgl. etwa Charlotte Bunch, A Feminist Human Rights Lens on Human Security, 2004, http://www.cwgl.rutgers.edu/globalcenter/charlotte/humansecurity.pdf (5.6.2008).

20 Vgl. den Bericht von Stephen Lewis, ehem. UN-Sondergesandter für AIDS in Afrika, I3.9.2007, http:// www.stephenlewisfoundation.org/news_speech_item.cfm?news=I 990 (20.1.2008).

2 I Mark Lacey, In Congo War, Even Peacekeepers Add to Horror, New York Times, I 8. I 2.2004, www.nytimes.com/2004/1 2/18/international/africa/1 8congo.html (3.6.2008).

22 Zum Kampf um Interpretationshegemonie Buckel (Fn. I4), 59, 60-62.

23 Karen Knop, Introduction, in: dies. (Hrsg.), Gender and Human Rights, Oxford 2004, I-I 2, I f.

24 Überblick zu rechtstheoretischen Verarbeitungen bei Sarah Elsuni, Feministische Rechtstheorie, in: Sonja Buckel/Ralph Christensen/Andreas Fischer-Lescano (Hrsg.), Neue Theorien des Rechts, Stuttgart 2006, I63-I85. 
Völkerrechtswissenschaft dar, die sich einer feministischen Menschenrechtspolitik verpflichtet fühlt. ${ }^{25}$

Interdependenzen betreffen die Ausdifferenzierung der Kategorie >Frau< im Hinblick auf andere zugeschriebene Merkmale. Dies bedeutet, Frauen, statt als einheitliche Gruppe, in ihren unterschiedlichen Lebenslagen wahrzunehmen, die sich aus dem Zusammenwirken von Geschlecht mit anderen Identitäts- bzw. Diskriminierungskategorien ergeben, etwa $>$ Rasse oder soziale Schicht. ${ }^{26}$ Die Kritik an einem weißen Mittelschicht-Alleinvertretungsanspruch wurde von den marginalisierten >Rändern $<$ her formuliert. ${ }^{27}$ Im Menschenrechtsdiskurs ist hier das Hierarchieverhältnis zwischen >Erste-, und >Dritte-Welt-Feministinnen $<\mathrm{zu}$ problematisieren. ${ }^{28}$ Dieses basiert zum Teil auf einer dichotomen (Selbst-)Konstruktion der emanzipierten weißen Frau gegenüber einer viktimisierten >Dritte-Welt-Frau< als kolonialem Erbe des feministischen Diskurses. ${ }^{29}$ Doch auch die Binarität der Geschlechter selbst, d.h. die strikte und auf Dauer gestellte Unterscheidung von männlich und weiblich ist fragwürdig geworden. Zunehmend akzeptiert ist, dass das soziale Geschlecht im Sinne von Rollenverhalten und Geschlechtsidentität (gender) vom biologischen Geschlecht (sex) unabhängig ist. Ein Denken in abgrenzbaren Körpern jedoch bietet immer wieder Anknüpfungspunkte für eine Trennung zwischen Männlichkeit und Weiblichkeit. Die Phänomene der Inter- und Transsexualität, die durch das kulturelle System der Zweigeschlechtlichkeit erst hervorgebracht werden, ${ }^{30}$ zeigen jedoch überdeutlich, dass auch die klar abgegrenzte biologische Zweigeschlechtlichkeit ein soziales Konstrukt ist. Im Lichte dieser Erkenntnis ist auch das politische, und damit das Rechts-Subjekt $>$ Frau< nicht mehr klar fassbar. ${ }^{3 \mathrm{I}}$

In einer heteronormativen Geschlechterordnung werden die binären Kategorien zudem in spezifischer Weise aufeinander bezogen. Das (konstruierte) biologische Geschlecht weist jeder Person nicht nur ein soziales Geschlecht zu, sondern auch ein normiertes Begehren. Ein sechter Mann< begehrt Frauen, eine sechte Frau< begehrt Männer (oder besser: nur einen Mann). ${ }^{32}$ Diese Ordnung ist auch

25 Zur Problematik des »borrowing « feministischer Theorie aus nationalen Kontexten für die Ebene des internationalen Rechts Murphy (Fn. 8), 77-82.

26 Kimberlé Crenshaw, Demarginalizing the Intersection of Race and Sex: A Black Feminist Critique of Antidiscrimination Doctrine, Feminist Theory and Antiracist Politics, in: Katharine T. Bartlett/Rosanne Kennedy (Hrsg.), Feminist Legal Theory: Readings in Law and Gender, Boulder/San Francisco/Oxford I991, 57-80 (Nachdruck von 1989) steht für den Begriff der Intersektionalität, während Cornelia Klinger/Gudrun-Axeli Knapp, Achsen der Ungleichheit - Achsen der Differenz: Verhältnisbestimmungen von Klasse, Geschlecht, >Rasse//Ethnizität, in: dies./Birgit Sauer (Hrsg.), Achsen der Ungleichheit: Zum Verhältnis von Klasse, Geschlecht und Ethnizität, Frankfurt am Main 2007, I9-4I von sich überschneidenden Achsen der Ungleichheit ausgehen; Katharina Walgenbach, Gender als interdependente Kategorie, in: dies. et al. (Hrsg.), Gender als interdependente Kategorie: Neue Perspektiven auf Intersektionalität, Diversität und Heterogenität, Opladen/Farmington Hills 2007, 23-64, 56 spricht demgegenüber von Gender als interdependenter Kategorie in komplexen strukturellen Dominanzverhältnissen.

$27 \mathrm{Zu}$ deutschen Genealogien Walgenbach (Fn. 26), $25-38$.

28 Dazu J. Oloka-Onyango/Sylvia Tamale, >The Personal is Political, < or Why Women's Rights are Indeed Human Rights: An African Perspective on International Feminism, Human Rights Quarterly I7 (1995), 69I-73I, 70I-705 und passim.

29 Chandra Talpade Mohanty, Under Western Eyes: Feminist Scholarship and Colonial Discourses, Feminist Review 30 (I988), 6I-88.

30 Zum Versuch der normierenden Inkorporation dieser Phänomene ins zweigeschlechtliche System vgl. Adrian de Silva (in diesem Heft). Zu den Widersprüchen in der Rechtsprechung ausführlich Laura Adamietz, Transgender ante portas? Anmerkungen zur fünften Entscheidung des Bundesverfassungsgerichts zur Transsexualität (BVerfG, Beschluss vom 6.I 2.2005 - I BvL 3/03), Kritische Justiz 2006, 368 380.

3 I Judith Butler, Das Unbehagen der Geschlechter, Frankfurt am Main I991, I 5-22 und passim.

32 Butler (Fn. 3I), 37-49; Adrienne Rich, Zwangsheterosexualität und lesbische Existenz, in: Elisabeth List/ Herlinde Studer (Hrsg.), Denkverhältnisse: Feminismus und Kritik, Frankfurt am Main i989, 244-280. Vgl. a. Julia König (in diesem Heft). 
in das Recht eingeschrieben und wird durch das Recht gestützt, das auch heterosexuelle Verhaltensweisen präferiert. 33

Werden diese Herausforderungen ernstgenommen, kann kein einheitliches Rechtssubjekt $>$ Frau $<$ mehr vorausgesetzt werden. $>$ Geschlecht $<$ muss vielmehr als gesellschaftliche Struktur gedacht werden, die basierend auf Binarität und Heteronormativität an Menschen Erwartungen stellt, die diese zum Teil nicht erfüllen können oder wollen, weil sie sich in ihrer Körperlichkeit oder ihrem Verhalten nicht eindeutig zuordnen wollen. ${ }^{34}$ In diesem Verständnis von Geschlecht laufen die bisher unterschiedenen Diskriminierungskategorien von Geschlecht im Sinne von `sex<, Geschlechtsidentität und sexueller Orientierung zusammen. 35

\section{Interdependenz, Binarität und Heteronormativität in der Analyse sexualisierter Gewalt in Kriegen}

Auf diese Weise zeigt sich, dass auch sexualisierte Gewalt in Kriegen analytisch differenzierter gefasst werden kann. Während die Anerkennung sexualisierter Gewalt gegen Frauen als Kriegsverbrechen und Fluchtgrund ein großer Fortschritt ist, fasst sie allein Gewalt gegen Frauen als geschlechtsspezifisch. Hierbei wird außer Acht gelassen, dass sich im Krieg spezifische Männlichkeiten und spezifische Weiblichkeiten konstituieren, die an gesellschaftliche Denkformen aus Friedenszeiten anknüpfen. Denn sexualisierte Gewalt in Kriegszeiten ist durchaus kein plötzlicher Einbruch, sondern Teil eines »Kontinuums der Gewalt «, die vor Ausbruch des Krieges beginnt und mit einem Friedensvertrag keineswegs endet. ${ }^{6}$

In Anknüpfung an patriarchale Besitzzuweisungen von Frauen an Männer werden Frauen in Kriegszeiten zum Symbol für die >Reinheit< der (ethnischen) Gemeinschaft und damit Schutzgut aller Männer, Männer werden zu Verteidigern ebendieser Gemeinschaft und ihrer Besitztümer.37 Im Rekurs auf diese Krieger-Männlichkeit ist die Vergewaltigung von Frauen damit eine >Kommunikation zwischen

33 Eingehend Jutta Hartmann/Christian Klesse/Peter Wagenknecht/Bettina Fritzsche/Kristina Hackmann (Hrsg.), Heteronormativität: Empirische Studien zu Geschlecht, Sexualität und Macht, Wiesbaden 2007. Zur deutschen Privilegierung der Ehe Laura Adamietz, Diskriminierung von Lebenspartnerschaften causa non finita. Anmerkung zu BVerfG, Kammerbeschluss vom 20.09.2007 - 2 BvR 855/06, STREIT 2008, i.E. Zu Heteronormativität und Zweigeschlechtlichkeit im Recht Adrian de Silva (in diesem Heft).

34 Gleichheitsrechtlich ansetzend Adamietz (Fn. 30), 379 f. und wohl auch Anne Koch-Rein, Geschlecht als Recht, Geschlecht als umfassend problematische Norm, STREIT 2006, 9-16; freiheitsrechtlich Andrea Büchler/Michelle Cottier, Intersexualität, Transsexualität und das Recht. Geschlechtsfreiheit und körperliche Integrität als Eckpfeiler einer neuen Konzeption, Freiburger FrauenStudien I7 (2005), I I 5-I40. Susanne Baer, Sexuelle Selbstbestimmung? Rechtsvergleichende Perspektiven zum Schutz vor Diskriminierung, in: Deutsches Institut für Menschenrechte (Hrsg.), Sexuelle Selbstbestimmung [Arbeitstitel], Berlin 2008, i.E., betont dagegen den Zusammenhang von Freiheit, Gleichheit und Menschenwürde (dazu ausf. dies., Dignity - Liberty - Equality. Aspects of a European Constitutional Triangle, Toronto Law Journal (2008), i.E.).

35 So auch Sarah Elsuni, Zur ReProduktion von Machtverhältnissen durch juridische Kategorisierungen am Beispiel >Geschlecht<, in: Lena Behmenburg et al. (Hrsg.), Wissenschaf(f)t Geschlecht: Machtverhältnisse und feministische Wissensproduktion, Frankfurt am Main 2007, I33-I47, I 43.

36 Cynthia Cockburn, The Continuum of Violence: A Gender Perspective on War and Peace, in: Wenona Mary Giles/Jennifer Hyndman (Hrsg.), Sites of Violence: Gender and Conflict Zones, Berkeley 2004, 24-44; Susanne Zwingel, Was trennt Krieg und Frieden? Gewalt gegen Frauen aus feministischer und völkerrechtlicher Perspektive, in: Cilja Harders/Bettina Roß (Hrsg.), Geschlechterverhältnisse in Krieg und Frieden: Perspektiven der feministischen Analyse internationaler Beziehungen, Opladen 2002, 175I 88, I78 f.; Anu Pillay, Violence Against Women in the Aftermath, in: Sheila Meintjes et al. (Hrsg.), The Aftermath: Women in Post-Conflict Transformation, London/New York 2001, 35-45.

37 Charlesworth/Chinkin (Fn. 2), 250-255. 
Männern $3^{8}$ in der die unterlegenen Männer symbolisch entmännlicht werden. Das Gefährdungspotential der jederzeit mobilisierbaren Krieger-Männlichkeit bringt aber auch Männer selbst in Lebensgefahr. Nicht nur Vergewaltigungen von Frauen, auch Tötungen von Männern sind daher geschlechtsspezifische Gewalt.

Die hegemoniale aggressive Männlichkeit39 konstituiert sich nicht nur binär im Verhältnis zur Weiblichkeit, sondern grenzt sich auch gegen marginale Formen von Männlichkeit ab, die ihrerseits feminisiert und homosexualisiert sind. Besonders plastisch zeigt sich dies am Beispiel des Falles Tadić:40

[The victim], who was naked and bloody from beating, was made to jump into the pit $[\ldots]$ and Witness $\mathrm{H}$ was ordered to lick his naked bottom and $\mathrm{G}$ to suck his penis and then to bite his testicles. Meanwhile a group of men in uniform stood around the inspection pit watching and shouting to bite harder. All three were then made to get out of the pit onto the hangar floor and Witness $\mathrm{H}$ was threatened with a knife that both his eyes would be cut out if he did not hold [the victim]'s mouth closed to prevent him from screaming; $G$ was then made to lie between the naked [victim]'s legs and, while the latter struggled, hit and bite his genitals. G then bit off one of [the victim]'s testicles and spat it out and was told he was free to leave. [... The victim] has not been seen or heard of since.

Das Machtgefälle zwischen Tätern und Opfern gestaltet die Übergriffe für die Opfer als homosexuell, ihre Feminisierung bestärkt die hegemoniale Männlichkeit der Täter als heterosexuell.4 Dies zeigt, wie sehr die Heteronormativität in die binären Geschlechterkonzepte eingeschrieben ist. Der Akt der nicht nur symbolischen, sondern tatsächlichen Kastration rekurriert zudem ganz explizit auf die Unterscheidung zweier auch körperlich grundverschiedener Geschlechter.

Männlichkeiten und Weiblichkeiten konstituieren sich dabei interdependent mit Kategorien wie Religion, Alter oder >Ethnie . Ein zentraler Ansatzpunkt der >ethnischen Säuberung durch Vergewaltigung bosnischer Musliminnen war eine religiös überformte >reine< Weiblichkeit; als besonderer Tabubruch in Sierra Leone galt die Vergewaltigung alter Frauen oder kleiner Mädchen, die der Sexualisierung schon oder noch entzogen waren..$^{2}$ In ethnisierten Konflikten gelten Männer nicht nur als Verteidiger der Gemeinschaft, sondern auch als biologische Träger der Ethnie, während die Ethnizität von Frauen von einem anderen Mann absorbiert werden kann. Damit genügt die gewaltvolle Inbesitznahme von Frauen, Männer dagegen müssen getötet werden, um das Fortbestehen der Ethnie zu verhindern. 43 Nicht nur Vergewaltigung und Kastration, auch das >banale< Töten ohne jegliche Berührung des Körpers/der Geschlechtsteile kann insofern sexualisiert sein, als es auf die spezifische männliche Funktion bei der Reproduktion rekurriert. Nicht nur Frauen, auch Männer sind somit wegen ihrer spezifischen Vergeschlechtlichung durch Kriegsgewalt bedroht.

38 Ruth Seifert, Krieg und Vergewaltigung: Ansätze zu einer Analyse, in: Alexandra Stiglmayer (Hrsg.), Massenvergewaltigung: Krieg gegen die Frauen, Frankfurt am Main I993, 94.

39 Cynthia Cockburn, The Gendered Dynamics of Armed Conflict and Political Violence”, in: Caroline O.M. Moser/Fiona C. Clark (Hrsg.), Victims, Perpetrators or Actors: Gender, Armed Conflict and Political Violence, London/New York 200I, I3-29, I6, 26; Cynthia H. Enloe, Maneuvers: The International Politics of Militarizing Women's Lives, Berkeley 2000, i 5 If.

40 ICTY Trial Chamber II, Prosecutor v. Dušan Tadić aka »Dule«, Case No. IT-94-1, Judgement, 7.5.1997, Rn. 206, http://www.un.org/icty/tadic/trialc2/judgement/index.htm (18.5.2008).

4I Joshua S. Goldstein, War and Gender: How Gender Shapes the War System and Vice Versa, Cambridge 200I, 359; Dubravka Zarkov, The Body of the Other Man: Sexual Violence and the Construction of Masculinity, Sexuality and Ethnicity in Croatian Media, in: Moser/Clark (Fn. 39), 69-82; Sandesh Sivakumaran, Sexual Violence Against Men in Armed Conflict, EJIL I8 (2007), 253-276, 270-273; R. Charli Carpenter, Recognizing Gender-Based Violence Against Civilian Men and Boys in Conflict Situations, Security Dialogue 37 (2006), 83-103, 94-96; Michael Meuser, >Doing Masculinity<-Zur Geschlechtslogik männlichen Gewalthandelns, in: Regina-Maria Dackweiler/Reinhild Schäfer (Hrsg.), Gewaltverhältnisse: feministische Perspektiven auf Geschlecht und Gewalt, New York/Frankfurt am Main 2002, 53-78.

42 Human Rights Watch, We'll kill you if you cry: Sexual Violence in the Sierra Leone Conflict, I5 (2003), $35 \mathrm{f}$.

43 Zarkov (Fn. 4I), 78; Sivakumaran (Fn. 4I), 274 f. 
Gesellschaftliche Verständnisse von Männlichkeit und Weiblichkeit sind daher

Grundlage der sexualisierten Gewalt in Kriegszeiten. Diese beruhen auf binären und heteronormativen Geschlechterkonzepten, die sich interdependent mit anderen Kategorisierungen ausformen.

\section{4. "Paradoxien einer Politik der Rechte«: Recht und Festschreibung von Kategorien}

Recht zum Schutz vor solcher Gewalt jedoch aktiviert die Kategorie Geschlecht. Auch Recht gegen Diskriminierung44 knüpft traditionell an Diskriminierungsmerkmale an und bestätigt damit symbolisch die Existenz eben der Kategorien, an die die Diskriminierung anknüpft. Das Bestehen auf der Gleichheit zweier Kategorien schreibt durch die Kategorisierung den Unterschied weiter fest. Diese Zweischneidigkeit wird bereits vom klassischen feministischen Dilemma benannt ${ }^{45}$ und gilt nicht nur für >sex<, sondern auch für >sexuality<.46 Der Kampf um Rechte erzeugt eine Identitätskategorie, die ihrerseits durch die Abgrenzbarkeit wieder einengend wirken kann. Appiah bezeichnet die Identitätsfixierung durch die Anerkennung von außen als »Medusa-Phänomen «.47 Recht schafft so zum einen Freiheiten, wirkt aber andererseits auch disziplinierend, als Normalisierungsmechanismus. $4^{8}$

Recht, das sich affirmativ auf >Frauen< und >Männer< beruft, wirkt daher mit an der Fortschreibung und Festschreibung von binären und heteronormativen Konstruktionen von Geschlecht als gesellschaftlicher Denkform, die ihrerseits der Nährboden für sexualisierte Gewalt ist - ein Paradoxon der Politik der Rechte, wie Hark formuliert.49 Ein Recht, das nicht teilhaben will etwa an der sexualisierten Gewalt gegen Frauen und Männer in Kriegen, müsste sich daher von einer binären, heteronormativen Kategorie >Geschlecht< lösen.

\section{Strategischer Essentialismus}

Doch kann das politische und das Rechts-Subjekt >Frau< wirklich (schon) aufgegeben werden? Die bereits erreichten Fortschritte zum Schutz von Frauen im Bereich des internationalen Rechts sind prekär und limitiert. Ein Ende des Redens von >den Frauen und ihrer >spezifischen Betroffenheit< könnte daher nicht zur erhofften Differenziertheit, sondern zu einer erneuten De-Thematisierung vergeschlechtlichter Unterdrückung und Gewalt führen. Hinzu kommt, dass nicht nur hegemoniale gesellschaftliche Denkformen, sondern auch die materielle Funktionalität der spezifisch vergeschlechtlichten Gesellschaftlichkeit

44 Zum Diskriminierungsschutz als Schutz vor hierarchisierender sexualisierter Gewalt Susanne Baer, Würde oder Gleichheit? Zur angemessenen grundrechtlichen Konzeption von Recht gegen Diskriminierung am Beispiel sexueller Belästigung am Arbeitsplatz in der Bundesrepublik Deutschland und den USA, Baden-Baden 1995, I90-244.

45 Martha Minow, Making All the Difference: Inclusion, Exclusion, and American Law, Ithaka, NY I990, 20 (»dilemma of difference ); detaillierter Gudrun-Axeli Knapp, Dezentriert und viel riskiert: Anmerkungen zur These vom Bedeutungsverlust der Kategorie Geschlecht, in: dies./Angelika Wetterer (Hrsg.), Soziale Verortung der Geschlechter: Gesellschaftstheorie und feministische Kritik, Münster 2002, I 5-62, 43.

46 Sabine Hark, Durchquerung des Rechts. Paradoxien einer Politik der Rechte, in: dies. et al. (Hrsg.), Queering Demokratie. Sexuelle Politiken, Berlin 2000, 28-44, Vorabdruck als Ohne Geländer handeln: Paradoxien einer Politik der Rechte, STREIT i 999, 59-68.

47 Kwame Anthony Appiah, The Ethics of Identity, Princeton 2005, 105-I I0.

48 S. dazu auch Elisabeth Holzleithner (in diesem Heft). Vgl. a. Elsuni (Fn. 35), I 35 -I 40.

49 Hark (Fn. 46). 
im kapitalistisch-arbeitsteiligen Produktionszusammenhang die Unterdrükkungsstrukturen Heteronormativität, Zweigeschlechtlichkeit und Patriarchat am Leben erhält. Gesellschaftliche Denkmuster werden daher nicht nur durch die Abbildung im Recht stabilisiert, sondern basieren auch auf materiellen Strukturen, die weder durch die Schaffung von Rechten noch die Abschaffung von Kategorien verschwinden. ${ }^{\circ}$ So bewirkt auch eine Ent-Biologisierung der Rechtskategorie >Rasse $<$ kein Ende des Rassismus. ${ }^{\text {I }}$

Eine Dekonstruktion des Subjekts >Frau < wird daher auch von intersektional differenziert denkenden Feministinnen wie MacKinnons2 oder Charlesworths3 abgelehnt. Auch afrikanische Feministinnen wie Tamale und Oloka-Oyango, die die Zweischneidigkeit des Rechts auch vor dem kolonialen Hintergrund diskutieren, wenden sich gegen »the post-everything, completely deconstructed and totally alienated nonreality in which western society is currently mired « 54 und argumentieren, »a united front is essential for any social movement. «s5 Ein >Rechte-Defätismus < ist in dieser Perspektive ein Luxus, den sich die leisten können, die bereits empowered sind.

Als Ausweg schlägt etwa Spivak einen >strategischen Essentialismus ${ }^{6} 6$ vor, der in der Analyse ohne eine abgrenzbare Kategorie >Geschlecht<, in der Rechtspolitik jedoch mit einem strategischen Bezug auf ein Rechtssubjekt >Frau< arbeitet. Butler, die den ganzen gender trouble mit ausgelöst hat, bezieht hier eine entspannt pragmatische Position: »Der Feminismus braucht >die Frauen muss nicht wissen, >wer< sie sind. «\$7

Diese strategische Position eröffnet auch die Möglichkeit der Allianz zwischen den Feminismen verschiedener >Generationen<, einer Gleichzeitigkeit des Ungleichzeitigen. Während genealogische Darstellungen feministischer Theorieentwicklungen oft eine Abfolge vom liberalen über den Differenz- zum dekonstruktivistischen Feminismus als chronologische und teleologische Entwicklung darstellen, gilt es mit Knapp, diese >Generationen $<$ eher als verschiedene >Räume des Feminismus zu verstehen, zwischen denen die Türen offen zu halten sind. $5^{8}$

\section{Choose your struggles}

Für eine zeitgenössische feministische Menschenrechtspolitik bedeutet dies, in der Analyse von und in der Politik gegen Menschenrechtsverletzungen struktu-

so Carol Smart, Feminism and the Power of Law, London/New York 1989, I44. Anstoß zur CLS-Kritik der Rechte in Mark Tushnet, An Essay on Rights, Texas Law Review 62 (1984), I363-I 403; kritischer Überblick in David Kennedy, The Critique of Rights in Critical Legal Studies, in: Brown/Halley (Fn. 7), I78-228.

รI Im Gegenteil hat das Tabu, das in Deutschland aus guten Gründen den >Rasse-Begriff umgibt, unter Umständen zu einer rechtlichen Unterbelichtung von Rassismus beigetragen. Vgl. Barskanmaz (in diesem Heft).

52 Catharine A. MacKinnon, Points Against Postmodernism, Chicago-Kent Law Review 75 (2000), 687-7 2.

53 Hilary Charlesworth, Feminist Methods in International Law, AJIL 93 (I999), 379-394, 383-385.

54 Oloka-Onyango/Tamale (Fn. 28), 723.

55 Ebd., 697.

56 Vgl. Gayatri Chakravorty Spivak, In Other Worlds: Essays in Cultural Politics, New York/London I 988, 205; vgl. a. Isabell Lorey, Dekonstruierte Identitätspolitik. Zum Verhältnis von Theorie, Praxis und Politik, in: Antje Hornscheidt et al. (Hrsg.), Kritische Differenzen - Geteilte Perspektiven. Zum Verhältnis von Feminismus und Postmoderne, Opladen I998, 93-I I4, I08.

57 Interview mit Judith Butler, Ort der politischen Neuverhandlung. Der Feminismus braucht >die Frauen<, aber er muss nicht wissen, >wer< sie sind, Frankfurter Rundschau, 28.3.2002.

58 Gudrun-Axeli Knapp, Macht und Geschlecht, in: dies./Angelika Wetterer (Hrsg.), Traditionen Brüche. Entwicklungen feministischer Theorie, Freiburg 1992, 287-331; Johannes Dingler/Regina Frey/Ute Frietsch/Ingrid Jungwirth/Ina Kerner/Frauke Spottka, Dimensionen postmoderner Feminismen. Plädoyer für Mehrstimmigkeit im feministischen Theorienkanon, Feministische Studien (2000), I29-I 44. Für diesen Hinweis und viele weitere Anregungen danken wir Aline Oloff. 
relle Dominanzverhältnisses9 in ihrer Komplexität und mit ihren materiellen Grundlagen in den Blick zu nehmen, jedoch nicht, an einer dieser Fronten erreichte Fortschritte leichthin aufzugeben und Rechtsinstrumente über Bord zu werfen, die sich auf die Kategorie >Geschlecht< stützen. Stattdessen muss auf die jeweilige Situation bezogen ausgehandelt und entschieden werden, wie der Geschlechtsbezug zu fassen ist.

Geschlechtsspezifische Menschenrechtsverletzungen etwa erscheinen, wenn Geschlecht in der Sachverhaltsanalyse differenziert mitgedacht wird, oft in einem allgemeineren Zusammenhang, der nur geschlechtsspezifisch überformt ist. Ist im ethnischen Krieg Vergewaltigung von Frauen das Pendant zur geschlechtsspezifischen Ermordung von Männern, kann etwa in der flüchtlingsrechtlichen Analyse Geschlecht als das >Partikulare< wieder zurücktreten hinter der ethnisch begründeten Verfolgung von Frauen und von Männern.60 Ein Bezug auf das Geschlecht erleichtert die Analyse, ist jedoch in der rechtlichen Einordnung schließlich nicht mehr notwendig; das scheinbar Partikulare wird zum Universalen.

Allerdings zeigt die Erfahrung auch, dass in universell formulierten Instrumenten Marginalisierte schnell >verschwinden<. Deshalb kann es im Einzelfall strategisch wichtig sein, durch entsprechende klarstellende Zusätze oder eigene Instrumente dieser Ausblendung entgegenzuwirken. Wird aus diesem guten Grund >Geschlecht` weiterhin genannt, kann jedoch durch die Einbeziehung von Geschlechtsidentität und sexueller Orientierung und ein interdependentes Geschlechtsverständnis einer Verfestigung repressiver essentialistischer Geschlechterverhältnisse entgegengewirkt werden. ${ }^{61}$

Eine Herangehensweise, die Geschlecht nicht als Identitätskategorie, sondern als gesellschaftliches Herrschaftsverhältnis und dabei immer als interdependent, d.h. als in sich nicht nur >überschneidenden`, sondern integral zusammenhängenden gesellschaftlichen Herrschaftsverhältnissen denkt und das Menschenrechtssubjekt >Frau< damit als vielfältiges und mit vielen Stimmen (wider)sprechendes und aus unterschiedlichen Machtpositionen unterschiedliche Interessen artikulierendes Subjekt fasst, hat die Instrumente an der Hand, sachorientiert Bündnisse einzugehen, in der unterschiedliche Interessen in gemeinsame Forderungen münden können, die für einen bestimmten Moment ein bestimmtes Rechtssubjekt >Frau< konstituieren können - »bridges between women are built, not found. «62 Dies setzt voraus, dass die unterschiedlichen Machtpositionen der beteiligten Gruppen und Individuen reflektiert werden und ein gleichberechtigter Aushandlungsprozess der verschiedenen Interessen stattfindet, in dem nicht einzelne Stimmen zum Schweigen gebracht werden. ${ }^{63}$ Die verschiedenen Feminismen müssen daher nicht nur die Türen offen halten, sondern auch die Ohren.

59 Walgenbach (Fn. 26), 56.

60 Hierzu Nora Markard, Fortschritte im Flüchtlingsrecht? Gender Guidelines und geschlechtsspezifische Verfolgung, Kritische Justiz 2007, 373-390, 383-386.

6I Vgl. Baer (Fn. 34), Selbstbestimmung; James D. Wilets, Conceptualizing Private Violence Against Sexual Minorities as Gendered Violence: An International and Comparative Law Perspective, Albany Law Review, 60 (1997), 989-1050; für das Flüchtlingsrecht Nicole LaViolette, Gender-Related Refugee Claims: Explanding the Scope of the Canadian Guidelines, IJRL I9 (2007), I69-2 I4 und Markard (Fn. 60), 390.

62 Angela P. Harris, Race and Essentialism in Feminist Legal Theory, Stanford Law Review 42 (I990), 58 I616, 61 s; vgl. Elsuni (Fn. 24), i 83 .

63 Zum Aushandlungsprozess >kultureller Praktiken im universalistischen CEDAW-Menschenrechtsdiskurs differenziert Sally Engle Merry, Human Rights and Gender Violence: Translating International Law into Local Justice, Chicago 2006. 